\title{
Time-evolution scenarios for short-range depletion gels subjected to the gravitational stress
}

\author{
Eleonora Secchi, ${ }^{*}$ Stefano Buzzaccaro and Roberto Piazza* \\ By exploiting photon correlation imaging and ghost particle velocimetry, two novel optical correlation \\ techniques particularly suited to the investigation of the microscopic dynamics of spatially \\ heterogeneous samples, we investigate the settling and restructuring dynamics of colloidal gels \\ generated by short-ranged depletion interactions. Three distinct regions can be clearly set apart within \\ the liquid-liquid coexistence region of the phase diagram where gel formation is observed. When \\ depletion forces are barely sufficient to drive the system within the metastable region, an initial \\ disordered gel hosts the rapid nucleation of crystallites, which stress the gel structure until it fully \\ collapses, leading to the formation of a macroscopic colloidal crystal. For stronger attractive forces, two \\ distinct scenarios are observed, depending on the particle volume fraction $\phi_{0}$ of the original suspension. \\ At low $\phi_{0}$, the gel breaks after a short delay time into separate clusters, which rapidly settle until they \\ compact in a denser disordered phase. The latter eventually undergoes a slow compression, which is \\ accounted for by a poroelastic model where the microscopic gel dynamics is fully ruled by its \\ macroscopic deformation. Yet, it is the intermediate stage between cluster settling and final compaction \\ which displays very peculiar features, evidenced by anomalous settling profiles which are not shared, to \\ our knowledge, by any other sedimentation processes investigated so far. For larger values of $\phi_{0}$, gel \\ breaking is conversely suppressed, the structure undergoes a continuous compression that cannot be \\ explained by a poroelastic model, and the microscopic dynamics is characterized by logarithmic \\ correlation functions resembling those found for attractive glasses.
}

Received 7th February 2014 Accepted 2nd May 2014

\section{Introduction}

When the attractive forces induced by the presence of a high molecular weight additive acting as a depletant become strong enough, a colloidal suspension undergoes a liquid-liquid (L-L) phase separation that, if the depletion forces are sufficiently short-ranged compared to the size of the colloidal particles, is metastable with respect to crystallization. ${ }^{1,2}$ As for simple liquid mixtures, a colloid suddenly brought within the L-L miscibility gap undergoes a spinodal decomposition process, consisting of phase separation followed by a progressive coarsening of the two phases. However, spinodal decomposition usually gets arrested by the formation of a colloidal network, that is, of a disordered colloidal solid with a gel-like structure. ${ }^{3}$

An ideal experimental survey of the morphology, microscopic dynamics, and spontaneous evolution in time of the arrested phases is however hindered by the relevant effect of weight on these very weak solids. Driven by gravitational stresses, the pristine gel structures undergo indeed consistent settling and compaction effects, whose time-dynamics and final outcome may be widely different depending on the range and

CMIC, Dipartimento di Chimica, Materiali e Ingegneria Chimica, Politecnico di Milano, 20133 Milano, Italy.E-mail: eleonora.secchi@polimi.it strength of the depletion interactions, and on the particle concentration in the gel. Extensive experimental investigations performed in the past decade ${ }^{4-11}$ has allowed identification of two main distinctive features of depletion gels restructuring under gravity. First, settling and compaction do not necessarily take place at once: quite often, a quiescent period is observed during which no macroscopic structural change is detected. A recent confocal microscopy investigation of this peculiar "delayed collapse" effect $^{10}$ has highlighted the crucial role played by the spontaneous coarsening of the structure that originates from the arrested spinodal decomposition. Moreover, because the bond strength is of the order of the thermal energy, the gel network restructures in time, getting progressively coarser and weaker, until it cannot sustain its own weight anymore. The quiescent stage is then ruled by intrinsic restructuring mechanisms, with gravity playing little role, so that its duration may be widely different depending on the gel concentration and on the strength and range of the interactions. Second, after the quiescent period, settling and compaction of the gel can occur in two very different ways, either by an initial sudden collapse of the network, followed by a much slower compaction stage, or via a slow, uniform compression. Although these two distinct scenarios have been related to the range and strength of the potential, ${ }^{11}$ one of the 
purpose of this work is to show that both of them may occur for a single system under the same interaction conditions by simply tuning the solid content of the gel.

The distinction between fast collapsing and slowly compressing (also called "creeping") gels is based on the consideration of the dependence on time of the position of the meniscus separating the settling/compacting gel from a particle-devoid supernatant phase. However, the amount of information carried by these simple measurements is very limited. In fact, a much better characterization of the gel restructuring kinetics may be obtained from measuring both the full time-dependent inhomogeneous concentration profiles that build up during the settling process and, possibly, the local sedimentation velocity at a given settling stage. Moreover, it is interesting to investigate whether and how the microscopic Brownian dynamics of the gel is related to macroscopic settling. The application of a novel scattering technique, which will be discussed in what follows, has recently allowed performing a comprehensive investigation of the late compression stage, following the initial fast collapse of a weak depletion gel. This study provided evidence that restructuring processes may persist over a very long time, and display a complex and rather surprising kinetics, where the gel macroscopic strain rate and microscopic dynamics are strongly interrelated..$^{8}$ It is useful to point out that these extremely slow restructuring processes make gravity effects important over long time scales, even for colloidal particles which are approximately density-matched with the suspending fluid. Besides scientific interest, understanding and possibly controlling gravity effects on colloidal gels is also crucial for improving the shelf lifetime of a large number of products addressed to the consumer and technical markets.

In this paper, we show that a detailed survey of the kinetics of gel settling and restructuring highlights several distinctive features that allow us to quantitatively relate the two main settling scenarios we have mentioned in the experimental phase diagram. By exploiting novel optical correlation and flow visualization methods, moreover, we shall show that widely different settling processes and flow profiles are associated with "collapsing" and "creeping" depletion gels. The first kind of gels, in particular, displays very peculiar kinetic sedimentation profiles, not reported so far for any other settling processes, which may yield useful hints about the relationship between these two gel classes. In addition, however, we also individuate a region within the $\mathrm{L}-\mathrm{L}$ gap where the gel structure that initially forms rapidly hosts the nucleation of colloidal crystallites, which grow until they lead to a collapse of the gel and to a fully crystalline equilibrium state. Similarities and differences between these final observations, which agree with numerical simulations by Fortini et al., ${ }^{12}$ and recent experiments on the formation of "crystal gels" under microgravity conditions ${ }^{13}$ will be discussed.

\section{Materials and methods}

\subsection{Colloidal system}

The colloidal systems used in this work are closely related to those we formerly investigated..$^{\mathbf{8 , 9 1 4}}$ In short, they consist of aqueous dispersions of spherical particles of MFA, a copolymer of tetrafluoroethylene and perfluoromethylvinylether, with a radius $R=90 \mathrm{~nm}$, a polydispersity of about $4 \%$ and a refractive index $n=1.356$. These particles display several features which make them suitable for the purpose of this investigation, namely, (i) their average refractive index is very close to that of water, hence the solvent and particle refractive indices can be matched by adding a small amount of urea, which makes light scattering studies fully feasible even for concentrated aqueous suspensions; (ii) they have a high material density $\left(\rho_{\mathrm{p}} \simeq 2.14 \mathrm{~g}\right.$ $\mathrm{cm}^{-3}$ ), which makes gravitational stress effects on the structures they form particularly relevant; (iii) they are partially crystalline, and therefore display depolarized scattering, which can be used as a very sensitive probe of sedimentation profiles. Depletion interactions are induced by the addition of the nonionic surfactant Triton X100, which forms globular micelles with a radius $r \simeq 3-4 \mathrm{~nm}$, corresponding to the size ratio $\delta=r / R$ $\simeq 0.03$. The surfactant also adsorbs on the particle surface forming a compact monolayer ${ }^{15}$ that provides steric stabilization even in the presence of about $0.1 \mathrm{M} \mathrm{NaCl}$, which we added to screen electrostatic interactions. In a former study, we showed that the equation of state (e.o.s.) of the MFA suspension, which can be tuned by varying the depletant volume fraction $\phi_{\mathrm{s}}$, closely conform to Baxter's Adhesive Hard Sphere (AHS), and can be used to map the experimental state points onto the theoretical phase diagram via virial coefficient matching. ${ }^{14}$ In the present study, the attention is strictly focused on the system behavior within the $\mathrm{L}-\mathrm{L}$ coexistence region, discussed in Section 3.

The suspensions, prepared at several values of particle and surfactant volume fraction (in the following respectively denoted as $\phi$ and $\phi_{\mathrm{s}}$ ), were poured into optical cuvettes with a square cross-section $5 \times 5 \mathrm{~mm}$, which were filled up to a height $h_{0}=$ $32 \mathrm{~mm}$. For preliminary qualitative assessments of the settling behavior, the samples are gently mixed and then kept steady at a room temperature of $23 \pm 1{ }^{\circ} \mathrm{C}$. We also prepared suspensions in both thinner $(3 \times 3 \mathrm{~mm})$ and wider $(10 \times 10 \mathrm{~mm})$ cells, and varied $h_{0}$ in the range $8-32 \mathrm{~mm}$, without detecting significant effects of the sample geometry on the location of the three gel classes in Fig. 2.

\subsection{Depolarized static light scattering (DSLS)}

As mentioned, the partial crystallinity of MFA particles yields a depolarized component in the scattered field which, similarly to what happens for incoherent neutron scattering, is insensitive to interparticle interactions and depends only on the local particle concentration. ${ }^{16}$ Hence, measurements of the depolarized scattered intensity, made by scanning along the vertical direction, allow reconstructing the inhomogeneous concentration profile induced by gravity settling. In our custom setup, the cell is mounted on a DC-motorized translation stage, allowing for an essentially vibration-free vertical positioning of the cell with $0.1 \mu \mathrm{m}$ resolution and an absolute reproducibility of $\pm 3 \mu \mathrm{m}$. A He-Ne laser beam is mildly focused in the cell to a beam waist of $46 \mu \mathrm{m}$, the incident and detected polarization states are accurately set by high-precision Glan-Thomson 


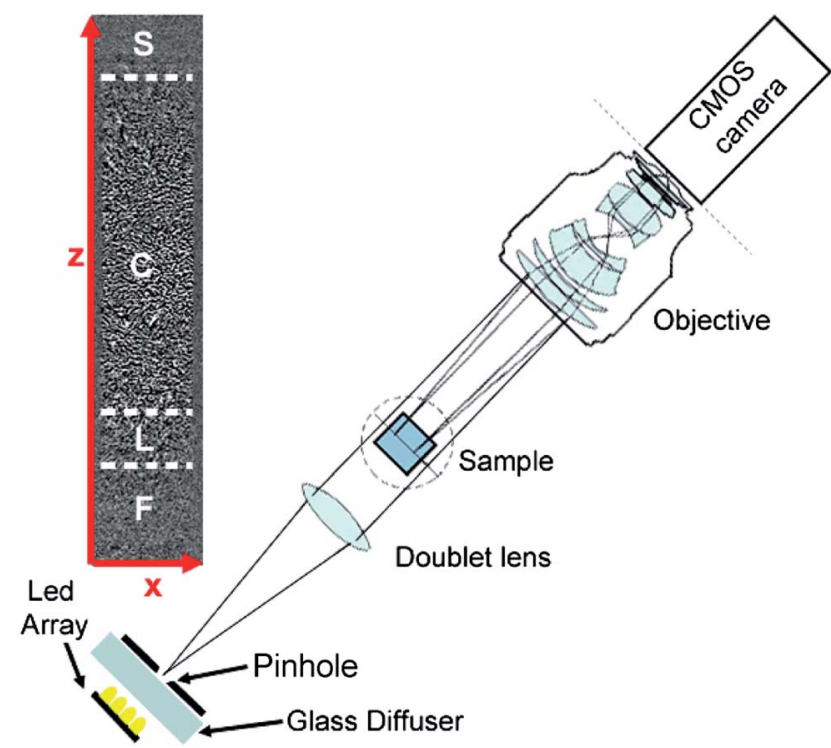

Fig. 1 Body: sketch of a GPV setup. Inset: typical speckle pattern obtained with GPV after background subtraction. On the top of the cell a clear supernatant (S) is visible, followed by a uniform column (C). The leg $(L)$ shows a reduction of the speckle pattern contrast that almost disappears in the foot (F). The $z$ and $x$ axes indicate the measurement coordinates.

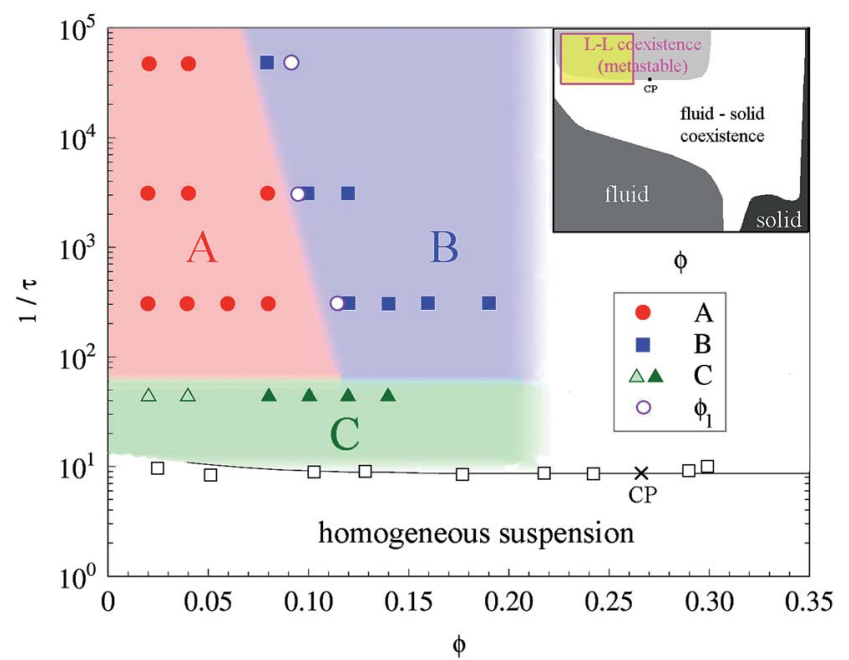

Fig. 2 State points for the three classes of depletion gels discussed in this work, plotted on a $(\phi, 1 / \tau)$ phase diagram (symbols, as indicated in the figure legend). The open squares show the experimental points where spinodal decomposition is first observed. These data are compared to the $L-L$ coexistence line for the AHS model obtained from numerical simulations (full line). ${ }^{24}$ The open circles indicate the approximate volume fraction $\phi_{1}$ of the peculiar "leg" feature observed in the settling profile of class A gels and discussed in Section 4 (the rectangle in the upper right inset shows the approximate location of the investigated region within a schematic phase diagram for a depletion system with the size ratio $\delta \simeq 0.03$ ).

polarizers with an extinction ratio better than $10^{-6}$, and the depolarized scattering intensity is measured at a fixed angle of $90^{\circ}$. The whole setup is enclosed in a removable case allowing us to control the temperature to better than $\pm 0.5{ }^{\circ} \mathrm{C}$. The absolute value of the local volume fraction at a given height $z$ is obtained by comparing the depolarized scattering intensity with the value obtained for a calibrated MFA suspension.

\subsection{Photon Correlation Imaging (PCI)}

$\mathrm{PCI}^{17}$ is a recently developed multi-speckle dynamic light scattering (DLS) technique conceptually similar to Time Resolved Correlation (TRC), ${ }^{18}$ but at the same time allows retaining the spatial resolution of an imaging system. Like TRC, it is designed to measure the slow and often intermittent time-dynamics of colloidal gels and glasses, which is precluded to standard DLS, but in addition it is particularly suitable to investigate spatially heterogeneous systems, such as a biopolymer solution undergoing a controlled gelation process. ${ }^{19}$

In our setup ${ }^{19}$ the sample, illuminated by a vertical laser sheet $(\lambda=650 \mathrm{~nm})$ with a thickness of $200 \mu \mathrm{m}$, is imaged by a lens on a CMOS sensor set at $\theta=90^{\circ}$ with respect to the illumination plane. Selection of a specific scattering wave-vector $q$ $=(4 \pi / \lambda) n \sin (\theta / 2) \approx 1.65 / R$ is obtained by suitably stopping down the numerical aperture of the collection optics with a diaphragm placed in the focal plane of the imaging lens. Besides defining $q$, the field diaphragm has the primary role of generating a speckle pattern on the image plane, due to the finite extent of the point spread function obtained by imaging through a stopped-down collection optics. The analysis of the images collected by the CMOS camera at a proper frame rate first consists in grouping together a suitable block of adjacent pixels (usually called a Region of Interest), over which the signal average and standard deviation is calculated. Increasing the number of pixels in a single block yields of course a better statistics, but reduces the spatial resolution. Very often, however, the symmetry of the problem allows picking up a single relevant direction along which the physical property of interest varies: in this case, the averaging block can be safely taken as a thin strip along the perpendicular direction. The local dynamics in each single block can be quantified by evaluating the intensity correlation function between two images taken at $t$ and $t+\tau$ :

$$
g_{2}(\tau ; t, \mathbf{r})=\left\langle\frac{\left\langle I_{\mathrm{p}}(t) I_{\mathrm{p}}(t+\tau)\right\rangle_{\mathrm{r}}}{\left\langle I_{\mathrm{p}}(t)\right\rangle_{r}\left\langle I_{\mathrm{p}}(t+\tau)\right\rangle_{\mathrm{r}}}\right\rangle_{\delta t}
$$

where $I_{\mathrm{p}}$ is the scattered intensity on pixel $p,\langle\cdots\rangle_{\mathrm{r}}$ is the average over all pixels contained in a block centered in $\mathbf{r}$, and $\langle\cdots\rangle_{\delta t}$ is a time average over an interval $\delta t$ much shorter than the typical time scale of the investigated dynamic processes.

Because PCI is in fact an imaging technique, it also allows us to single out collective motion within the sample, namely, to detect the presence of hydrodynamic motion. This is particularly relevant for the present study: similarly to what happens in Particle Image Velocimetry (PIV), in PCI measurements the position of the cross-correlation peak between two speckle patterns obtained at different times is indeed directly related to local physical displacements in the sample. The amplitude of the correlation peak, moreover, decays on the time scale of the local rearrangement dynamics, allowing setting apart the spurious contribution to the decay of the correlation function due to the 
overall translation of the speckle pattern. This strategy, which has already been profitably exploited to investigate the long-time restructuring dynamics of colloidal gels, ${ }^{\mathbf{8}, 9}$ is effective only provided that the internal dynamics is sufficiently slow that the speckle fields remain correlated enough over the time it takes for the sample to display a detectable collective motion. This is not a problem in the final restructuring stage of a gel, when the local dynamics has strongly slowed down, but severely challenges the possibility of using PCI to study the initial collapsing stage of a gel into fast settling clusters, which we plan to investigate. Such a limitation could in principle be bypassed by collecting the scattered light at much smaller $q$-vectors, namely, by performing PCI in a small-angle light scattering (SALS) configuration: yet, a much simpler solution consists in using the different strategy described in the following Section.

\subsection{Ghost Particle Velocimetry (GPV)}

In the past few years, novel experimental configurations have allowed getting rid of several criticalities of a standard SALS setup. $^{20}$ The common strategy shared by these techniques is to collect the light scattered by the sample in "near field" (NF), which more technically means on a plane lying within the socalled "deep Fresnel" diffraction region. To see why this region is so interesting, it is useful to discuss the simple case of scattering from a dilute colloidal suspension. Whereas in the farfield region probed by a standard light scattering experiment the size of the speckles generated by the interference of the fields scattered by the particles is fixed only by the geometry of the scattering volume, providing no physical information on the sample, within the deep Fresnel region, which can be properly defined, ${ }^{21}$ the average size of a speckle coincides with the particle size, yielding therefore important information on the sample physical structure. In the more general case, the power spectrum of the intensity on a NF plane is directly related to the structure factor of a suspension. Then, a simple NF scattering experiment just consists of sending an expanded laser beam on the sample and collecting the optical signal with a CCD or CMOS camera placed along the optical axis at a short distance $z$ from the sample. Since this signal is the result of the superposition of the field $E_{\mathrm{tr}}$ associated with the transmitted beam (namely, the unscattered fraction of the incident field $E_{0}$ ) with the field $E_{\mathrm{s}}$ scattered at small angles, this is in fact a heterodyne scheme. As a consequence, the intensity pattern on the detection plane shows therefore a time-fluctuating speckle pattern, whose amplitude is modulated by the transmitted field, on top of a strong, but time-independent background $I_{\mathrm{tr}}=\left|E_{\mathrm{tr}}\right|^{2}$, which can be easily eliminated by subtracting out the intensity pattern averaged over a sufficiently long time. The fluctuation in time of each single speckle is related to the microscopic Brownian dynamics of the sample as a superposition of the decay modes for all the detected $q$-vectors, which can be set apart by a Fourier analysis yielding the correlation function at each $q$. Conversely, the time-displacement of the speckle pattern mirrors the local collective motion within the sample, allowing measurement of a full hydrodynamic velocity pattern like in a PIV measurement, but with no need to add tracers.
The original NF approach exploits a laser source, but this is not strictly necessary. In fact, what is really required is a source with a sufficiently high degree of spatial coherence, and this can simply be obtained by using a diaphragm to limit the spatial extension of any monochromatic source such as a spectral lamp, the full spatial coherence of a laser corresponding to the limiting case of a point source. Monochromaticity is really not an issue either, since the speckle patterns corresponding to different wavelengths superimpose at sufficiently small scattering angle. These considerations have allowed Cerbino and Trappe ${ }^{22}$ to develop a simple but powerful technique, dubbed Differential Dynamic Microscopy (DDM), where the same information obtained in a NF experiment can be obtained using a standard microscope illuminated by a white-light source in a Köhler configuration, provided that the numerical aperture of the condenser is suitably reduced by stopping-down the field diaphragm.

Recently, we have introduced a novel technique, "Ghost Particle Velocimetry" (GPV), that combines the simplicity and convenience of DDM with the distinctive power of the NF measurements to allow for velocimetry measurements without the need for the addition of tracers. ${ }^{23}$ GPV proves to be very effective in obtaining the flow pattern in microfluidic devices from a PIV-inspired analysis of the images obtained with a simple microscope. Yet, the application of the concepts on which DDM and GPV are based is not confined to microscopy: in fact, the same ideas can be exploited for any optical setup where the illumination source, even if not monochromatic, has a sufficient spatial coherence. In the setup used in this investigation, illumination is provided by an array of high-power white-light LEDs, and smoothed out by a ground glass placed close to the array. The source is limited in size by a pinhole with a diameter $D \simeq 1 \mathrm{~mm}$ to enhance the degree of spatial coherence, and placed in the rear focus of an achromatic doublet, which turns it into a parallel beam with a diameter of about $50 \mathrm{~mm}$ and a transversal spatial coherence length of about $400 \mu \mathrm{m}, \dagger$ which illuminates the sample cell. A transmission image of the sample is formed on the CMOS camera by a photographic objective whose magnification can be tuned, which allows expanding the view of specific details within the sample (Fig. 1). In this work GPV will be mostly used to obtain more accurate velocity fields than feasible using PCIt or to analyze the microscopic dynamics of fast cluster settling.

Summing up, several pieces of information can be extracted from the analysis of GPV experiments:

1. Local motion within the sample can be qualitatively appreciated from the background-subtracted images (obtained, in the non-stationary case, either by high-pass filtering or by

$\dagger$ Positioning the source in the lens focal plane is equivalent to using "limiting" Köhler illumination with a conjugate plane placed at infinity, hence the longitudinal spatial coherence length $\ell_{\mathrm{c}}$ of the beam is large $\left(\ell_{\mathrm{c}}=c / \Delta \nu \simeq 1 \mathrm{~cm}\right.$, where $\Delta \nu$ is the temporal bandwidth of the LED source). This configuration lacks the power of obtaining spatial resolution along the optical axis, which characterized DDM and GPV measurements made with the partially coherent illumination provided by a microscope, but allows for a better contrast in the image of the speckle pattern.

\$ Because the sample is imaged at large $q$-vector, the speckle pattern fluctuates much faster, making PCI not suitable to detect slow settling motion. 
taking the difference between images separated by a sufficiently long delay).

2. A straightforward impression of how fast is the local microscopic dynamics in the sample at time $t$ is provided by an activity map, obtained from the standard deviation over small averaging blocks of the intensity difference between two images.

3. A quantitative evaluation of both the velocity field pattern and of the microscopic correlation functions can be obtained by a PIV-like analysis, by respectively considering the displacement and the intensity of the peak in the spatial cross-correlation of different images.

\section{The $L-L$ coexistence region: a general scenario for MFA depletion gels}

The equation of state and the full phase diagram for the MFA + Triton colloidal system in the presence of $100 \mathrm{mM} \mathrm{NaCl}$ have already been presented in a previous work, which shows that the system closely conform to the AHS model. ${ }^{14}$ It is then convenient to plot the coexistence region, which is the region of interest for the gel phases, using as variables the particle volume fraction $\phi$ and the reciprocal of Baxter's "stickiness" parameter $\tau$ that can be related to the depletant volume fraction $\phi_{\mathrm{s}}$ by fitting the experimental equations of state. $\S$ For convenience, the values of $\tau$ corresponding to the depletant concentrations of the samples investigated in this work are summarized in Table 1.

The coexistence curve (binodal line) for the AHS model obtained from numerical simulations is compared in Fig. 2 to the state points where phase separation is first observed (open squares), which can be found by detecting the increase of turbidity associated with the crossing of the spinodal line. 9 By quenching into the coexistence region by the addition of a further amount of depletant, a fast demixing of the suspension is observed. Unless the quench is extremely shallow, however, this phase separation process rapidly comes to a halt, and the system freezes into an amorphous solid structure, namely, a depletion gel, as clearly evidenced by a sudden dynamic arrest of the scattering speckle pattern.\|

$\S$ This quantity is simply related to the 2 nd osmotic virial coefficient $B_{2}$ as $\tau^{-1}=$ $4\left(1-B_{2} / B_{2}^{\mathrm{hs}}\right)$, where $B_{2}^{\mathrm{hs}}$ is the virial coefficient for hard spheres.

I In the flat region of the coexistence curve, the binodal and the spinodal lines (actually, a mean-field concept, which can nevertheless be defined as the limiting line where the compressibility diverges) basically coincide. As a matter of fact, for short-range attractive potentials the two lines are usually extremely close over most of the coexistence curve. ${ }^{25}$ We point out, however, that in the systems we investigated the turbidity increase is rather small, and is only due to the fact that index-matching is exact only at the wavelength of the laser used for GPV and PCI measurements. This gives an uncertainty on the position of the spinodal line of the order of $2 \%$.

$\|$ It is useful to stress again that, in depletion systems, the formation of amorphous solids from a dilute or moderately concentrated suspension requires the fast phase separation process occurring within the L-L coexistence gap. ${ }^{3}$ For instance, while a suspension already prepared at very high concentration may behave as a repulsive or attractive glass, ${ }^{26}$ a more dilute sample that slowly concentrates by sedimentation does not get arrested into a disorder solid, but rather reaches an equilibrium colloidal crystal phase having a concentration pretty close to the ordered close packing limit. ${ }^{14}$
Table 1 Stickiness parameter $\tau$ for several values of the volume fraction $\phi_{\mathrm{s}}$ of added surfactant

\begin{tabular}{ll}
\hline$\phi_{\mathrm{s}}$ & $\tau$ \\
\hline Spinodal line: $0.079-0.081$ & $0.10-0.12$ \\
0.10 & $2.2 \times 10^{-2}$ \\
0.12 & $3.3 \times 10^{-3}$ \\
0.14 & $3.3 \times 10^{-4}$ \\
0.16 & $2.0 \times 10^{-5}$
\end{tabular}

Nevertheless, as we already mentioned, in the presence of the gravitational stress these structures are mechanically unstable, and may display a diverse evolution in time depending on their location within the coexistence region, namely, on both the strength of the depletion forces (quantified by $\tau^{-1}$ ) and their initial particle volume fraction $\phi_{0}$. By preparing samples at several different values of $\phi_{0}$ and $\phi_{\mathrm{s}}$, we have identified three main classes of gels:

(A) Collapsing gels (red circles in Fig. 2): for sufficiently deep quenches $(0.1 \leqq \tau \lesssim 0.02)$ within the coexistence gap, but not too large values of $\phi_{0}$, the gels undergo a first rapid collapse, which is however followed by a much slower compacting stage with no crystal nucleation. Eventually, the system reaches a time-invariant profile which is characteristic of a dense colloidal gel.

(B) Creeping gels (blue squares in Fig. 2): for comparable quenches of samples prepared at a sufficiently larger initial concentration $\phi_{0}$, no gel breaking is conversely observed. Rather, the gel compacts smoothly, with a compression kinetics that is highly non-linear in time.

(C) Crystallizing gels: when the quench within the L-L gap is very shallow, the tenuous gels that initially form by spinodal decomposition may (open triangles in Fig. 2) or may not (full triangles) start to display a collapsing stage similar to that shown by class A gels. However, after a few tens of minutes, they host the nucleation of ordered crystallites, which grow until they disrupt the gel structure and eventually lead to the formation of a single AHS colloidal crystal.

A full characterization of the time evolution under gravity of the first two gel classes will be presented in Sections 4 and 5, whereas the crystallization processes observed for class $\mathrm{C}$ gels will be only given a preliminary qualitative description in Section 6.

\section{Class A: collapsing gels}

\subsection{Time-evolution of the total height of the gel}

"Collapsing" gels are generated by samples prepared at a depletant concentration $\phi_{\mathrm{s}}>0.1$, corresponding to a moderately deep quench into the $\mathrm{L}-\mathrm{L}$ gap, but at sufficiently low $\phi_{0}$, which gets the lower the deeper the quench. It is worth noticing that all the depletion gels we formerly investigated ${ }^{8,9}$ belong to this class. As we already mentioned, these gels display two rather well separated restructuring stages, which take place after a rather short initial quiescent state, whose duration $t_{\mathrm{d}}$ is rather erratic, but limited to less than an hour for all the samples we 
have investigated. After this delay period, the gel displays a first collapse stage, witnessed by the rapid descent of the meniscus separating the dense phase from the supernatant, followed by a much longer compacting stage showing a very different kinetics. This general behavior is exemplified in Fig. 3 for a gel prepared at $\phi_{0}=0.04$ in the presence of a surfactant volume fraction $\phi_{\mathrm{s}}=0.14$. As shown in the figure, during the collapsing state the meniscus falls linearly with time, which is what happens for a colloidal fluid settling at a constant sedimentation velocity. In this case, however, the settling speed $v_{\mathrm{s}} \simeq 6 \mathrm{~mm}$ $\mathrm{h}^{-1}$ is about two orders of magnitude larger than the sedimentation velocity of a dilute suspension of MFA particles, which strongly suggests that the settling objects are actually large particle clusters generated by sudden breaking of the gel.

After about 4 hours, the gel enters a compaction stage, whose kinetics and microscopic dynamics has already been extensively studied in our former investigations. ${ }^{8,9}$ In the following, with the aim of facilitating a comparison with class B gels, we shall just recall the main results of these studies, focusing more on the collapsing stage. Although in this stage the descent of the meniscus suggests a rather trivial sedimentation process, a detailed investigation of the full kinetic sedimentation profiles obtained by DeLS unravels indeed a much more surprising, and for several reasons puzzling, settling scenario.

\subsection{Sedimentation profiles during the collapse stage}

In order to appreciate better the very peculiar settling profiles observed during the collapse stage, it is worth recalling some general features of the kinetic sedimentation profiles shared by any colloidal fluids. While settling, the suspension, which at the

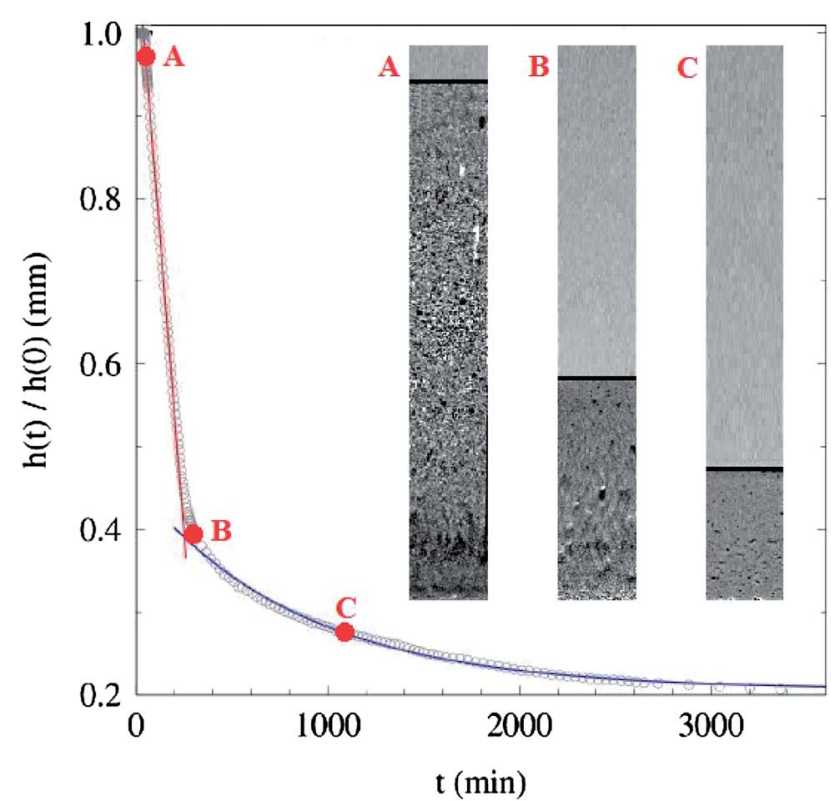

Fig. 3 Body: kinetics of the meniscus descent for a collapsing gel prepared at $\phi_{0}=0.04$ in the presence of a surfactant volume fraction $\phi_{\mathrm{s}}=0.14$. Insets: snapshots of the cell, obtained with GPV using the first method of background subtraction (see text for details), at three different times indicated by red points in the body of the figure. beginning displays of course a uniform concentration profile shaped as a vertical "column" at the constant volume fraction $\phi_{0}$, progressively generates a more concentrated "foot" (also know in the literature as a "cake") close to the cell bottom, leaving in the upper part of the cell a pure solvent phase (the supernatant) visually separated from the settling suspension by a meniscus that, at a closer inspection, actually consists of a thin "fan" where the particle concentration smoothly increases from 0 to $\phi_{0}{ }^{* *}$ Notably, for a colloidal fluid the whole region between the fan and the foot remains at constant uniform concentration $\phi_{0}$, progressively shrinking until the fan and the foot start to merge. Once this column has disappeared, the foot still evolves for a long time, eventually reaching the equilibrium concentration profile dictated by the equation of state, unless a kinetic arrest into a glass phase takes place.

Since the linear descent of the meniscus strongly suggests that the gel has fully broken into a cluster fluid, this is the behavior we may expect to see for class A gels in the collapsing stage, whatever the size and structural organization of the settling aggregates. Yet, this is not the case: the settling profile has a peculiar and unexpected shape characterized by the presence, just on top of the foot, of an additional region that is still uniform, but has a concentration $\phi_{1}$ significantly larger than $\phi_{0}$. Considering the fan as the "head" of the settling profile and the standard column its "torso", we may regard this unexpected region over the foot as a kind of "leg". The morphology of several of these "legs", observed for samples at $\phi_{0}=0.04$ and different values of $\phi_{\mathrm{s}}$, are shown in Fig. 4 . As evident, $\phi_{1}$ depends rather weakly on the strength of the depletion forces, although the values of $\tau$ cover about two orders of magnitude in the same range of $\phi_{\mathrm{s}}$.

An example of the full time-evolution of a settling profile is shown in Fig. 5. Because the region at $\phi=\phi_{1}$ settles at a much slower pace than the overhanging column, the clusters in the latter progressively catch up and accumulate on top of the leg, so that the boundary between the latter and the column actually rises in time, until the column supply runs out. The gel then enters the slow compaction stage during which the leg progressively merges with the foot, until a time-invariant profile is reached. This latter compaction phase has been shown to be fully accounted for by a poroelastic model. ${ }^{8,9}$

We have previously found ${ }^{9}$ that the shape of the profile eventually reached by the gel (which we shall conventionally call the "equilibrium" profile, although it does not reflect of course a state of real thermodynamic equilibrium) does not depend on the initial particle concentration: in fact, profiles obtained for different values of $\phi_{0}$ can be rescaled one on top of the other by a simple translation along the vertical axis. This suggests that the clusters forming upon gel breaking have a similar morphology regardless of the value of $\phi_{0}$, so that the gels they eventually generate by compacting and merging in the sediment have

** After some time, which may be very short for moderately concentrated suspensions, the fan takes on a time-invariant shape, dictated by the competition between Brownian diffusion and the self-sharpening effect brought in by the dependence on concentration of the sedimentation velocity, which is in fact a concentration "shock wave". ${ }^{27}$ 


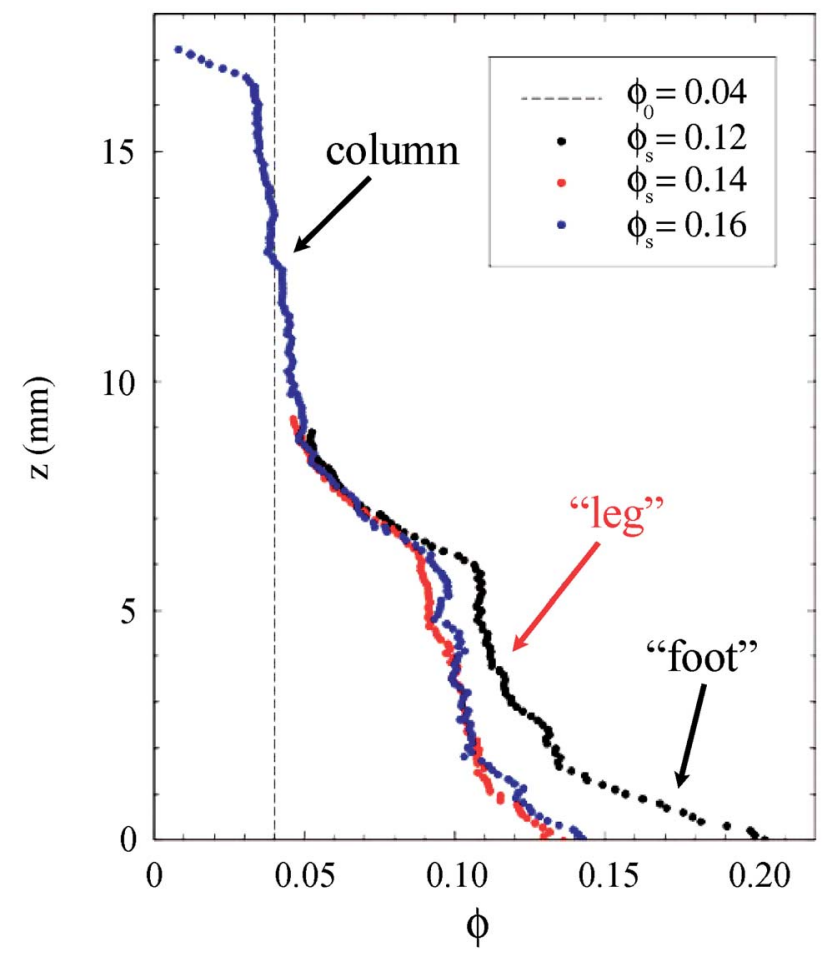

Fig. 4 Examples of the "leg" structures building up during the settling process of three different class A gels prepared at the same MFA volume fraction $\phi_{0}=0.04$ but for different values of $\phi_{\mathrm{s}}$.

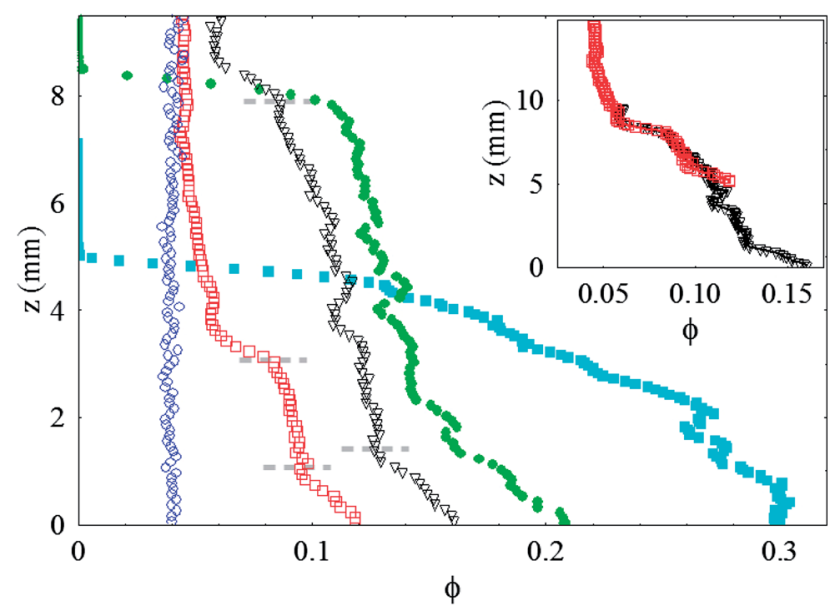

Fig. 5 Main Body: time-evolution of the settling profiles for a MFA suspension at $\phi_{0}=0.04$ in the presence of a Triton concentration $\phi_{\mathrm{s}}=$ 0.14 at $t=0 \mathrm{~h}$ (blue open circles), $t=1 \mathrm{~h}$ (red open squares), $t=3 \mathrm{~h}$ (black open triangles), $t=10 \mathrm{~h}$ (green dots), and $t=118 \mathrm{~h}$ (light blue squares). The leg region is approximately framed by the dashed lines. Inset: profile at $t=1 \mathrm{~h}$, vertically translated on top of the $t=3 \mathrm{~h}$ profile to show that the leg shape does not change with time.

similar elasto-mechanic properties. Conversely the equilibrium profiles corresponding samples with the same $\phi_{0}$ but different amounts of depletant do not rescale, since stronger attractive interactions lead to stiffer gels with a higher compression modulus.

\subsection{Speckle dynamics and velocity fields}

A GPV analysis of the velocity fields and of the local microscopic dynamics during each settling stage provides further information on the origin and nature of the distinctive "leg" structure we observed. Let us first discuss the gel breaking process qualitatively. A typical breaking sequence is displayed in the upper panel of Fig. 6, which shows five images of the upper region of a gel at $\phi_{0}=0.04, \phi_{\mathrm{s}}=0.14$ obtained by taking the difference, normalized to the average intensity, of pairs of frames taken $\Delta t=5 \mathrm{~s}$ apart. During the quiescent period $\left(t<t_{\mathrm{d}}\right)$, no detectable rearrangement of the gel is observed over $\Delta t$, hence the difference signal is negligible. At $t \simeq t_{\mathrm{d}}$ (first image), however, the beginning of the breaking process is highlighted by a substantial change within $\delta t$ of the speckle pattern in the two regions shown by the yellow arrows, indicating the onset of two vertical streams (second image) that originate close to the top of the sample and progressively extend downwards until they merge, approximately in the middle of the cell (third image). Afterwards, the breaking process becomes extremely fast, rapidly leading to the full disruption of the gel structure (last two images).

The local dynamics during gel breaking can be better quantified by plotting the activity maps for same values of $t$, obtained by evaluating the standard deviation $\sigma(\mathbf{r}, t)$ in a small

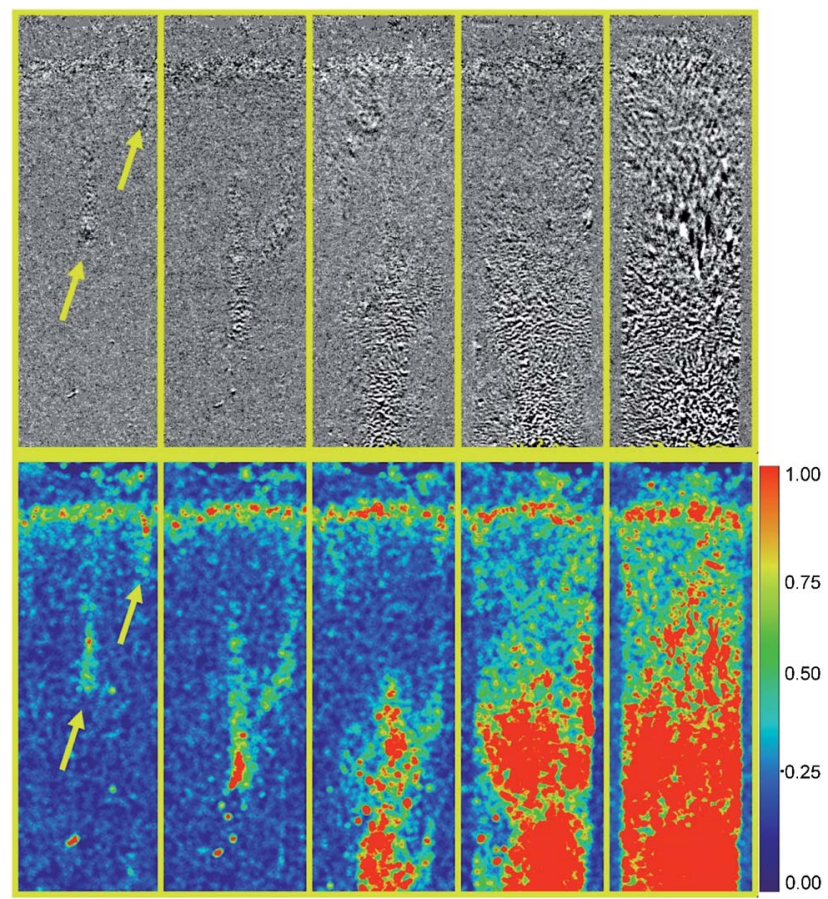

Fig. 6 Breaking dynamics of a MFA depletion gel at $\phi_{0}=0.04, \phi_{\mathrm{s}}=$ 0.14 . Top panel: GPV difference images obtained by subtracting frames taken $5 \mathrm{~s}$ apart. The first frames of the 5 displayed difference images were acquired at $t=18,21,24,27$, and $30 \mathrm{~min}$. Bottom panel: "Activity maps" at the same values of $t$, obtained by plotting in false color the normalized standard deviation, of the intensity within a circular region with a radius of 5 pixels around each location. The scale to the right displays the color scale for the normalized standard deviation. 
circular block around each position in the gel of the difference signal between two images acquired at $t$ and $t+\Delta t$, with $\Delta t=5 \mathrm{~s}$, which, as already discussed, gets the larger the more the two speckle fields get uncorrelated. The lower panel of 6 , where the progressive increase of $\sigma$ is shown in a color scale from blue to red, shows that, as expected, the regions where gel breaking develops show a much faster local dynamics. We wish to stress that, although the delay time $t_{\mathrm{d}}$ shows fluctuations from sample to sample, for all class A gels we investigate rupture which was found to start at or close to the upper meniscus. This implies that the gravitational stress (of course stronger close to the cell bottom, where the structure has to sustain the whole weight of the gel) is not the main factor responsible for gel breaking, and rather suggests that during the quiescent period the gel structure coarsens as a whole, in contrast with previous finding for other short range systems ${ }^{\mathbf{4 , 2 8 , 2 9}}$ but similarly to what described by Bartlett et al., ${ }^{7,10}$ until rupture is triggered by a weak perturbation, which is more likely to occur close to the curved meniscus between gel and air. To check this, we have prepared a sample with the same values of $\phi_{0}$ and $\phi_{\mathrm{s}}$, filling completely the cell and mounting it upside down, so that the upper gel interface coincides with the flat cell bottom, but deliberately leaving a few air bubbles within the cell. The buoyancy force acting on these bubbles is sufficiently strong that they, although tiny, slowly drift to the surface. Nevertheless, during the quiescent period, the bubbles just "creep" through the gel, which apparently manage to self-heal. Conversely, for $t>t_{\mathrm{d}}$, the bubble drift triggers the gel breaking, which begins in the bubble wakes and rapidly propagates to the whole structure. This catastrophic collapse contrasts with the behavior of gels with longer range attractive force, ${ }^{10}$ which apparently show a large number of independent microcollapses whose effects are localized in space, resulting in a meniscus that falls with $v(t) \propto t^{1 / 2}$. Because of a much less effective viscous damping, shorter-range attraction leads then to stress propagation over much larger distances, similarly to what has been observed in the PCI activity maps obtained for gels generated by irreversible aggregation. ${ }^{30}$

The speckle fields associated with the fast settling clusters fluctuate too rapidly to allow for a detailed characterization of the velocity field. Qualitatively, however, the latter is far from being laminar and initially shows a number eddies turning in random direction, which usually merge after a short time into a single roll involving the whole column. We recall however that, according to the DeLS profiles, the column remains uniform, which means that no appreciable coupling between velocity and concentration fluctuations is detected. Once the clusters merge into the leg region, the dynamics of the speckle field slows down considerably, and the velocity profile can be followed by GPV.

A close inspection of the dependence of the standard deviation of the GPV difference images allows rough identification of the "leg" detected in the concentration profiles as a region along the vertical axis $z$ where the local dynamics is considerably slower than in the overhanging column, but still much faster than in the almost arrested underlying foot. To see this, it is convenient to average the local standard deviation along the horizontal axis $x$ by defining rectangular blocks as wide as the cell, and with a thickness $\Delta z$ of 4 pixels. This smoothed and horizontally averaged standard deviation $\sigma(z, t)=\langle\sigma(\mathbf{r}, t)\rangle_{x, \Delta z}$ can then be plotted as a function of time as shown in Fig. 7, where the behavior of $\sigma(z, t)$ at each given time is then represented by a vertical line with the same colour code we formerly used. While settling proceeds, the sample shows a progressive buildup of an intermediate region between a highly dynamic column, where the speckle field fully rearranges within $5 \mathrm{~s}$ separating two successive frames, and an almost quiescent sediment (the foot), where conversely changes in the speckle field within this time scale are almost negligible. As a matter of fact, $\sigma(z, t)$ attains values within the leg region which are about $50 \%$ of its maximum, suggesting that local rearrangements take place on time scales of the order of tens of seconds. The black and the white dashed lines in Fig. 7 give an indication of the transitions between the column and the leg and, respectively, the leg and the foot, obtained by defining two threshold values $\sigma_{\mathrm{cl}}(z, t)=0.5 \sigma_{\mathrm{c}}(t)$ and $\sigma_{\mathrm{lf}}(z, t)=0.2 \sigma_{\mathrm{c}}(t)$, where $\sigma_{\mathrm{c}}(t)$ is the maximum value of the standard deviation within the column at time $t$. Consistently with the concentration profiles, the column gets progressively depleted, until at $t \simeq 25 \mathrm{~mm}$ it fully merges with a consistently thickened leg region.

Within the leg and foot regions, where the speckle dynamics is sufficiently slow, GPV also allows reconstruction of the $x$ averaged velocity profile $v_{z}(z)$ along $z$. Since the position of these regions changes with time, it is convenient to plot $v_{z}(z, t)$ by translating the $z$-axis so that $z=0$ is taken as the position at time $t$ of the leg/foot interface, as determined by the white broken line in Fig. 7. Fig. 8 shows that, with this device, all velocity profiles rescale on a single curve. The plot shows that $v_{z}(z)$, which is very small and roughly linear in $z$ in the foot region, grows by an order of magnitude within a thin transition region between the foot and the leg, to recover a linear trend, but with a larger slope within the leg. This means that the strain rate $\dot{\varepsilon}=\mathrm{d} \nu(z) / \mathrm{d} z$ is approximately constant in the bulk of both regions but, for the gel we are considering, about ten times smaller in the foot than in the leg, which consistently compresses much faster. Once the leg has fully merged with the foot, $\dot{\varepsilon}$ decreases almost exponentially with time, in agreement with the predictions of a poroelastic model. ${ }^{8,9}$

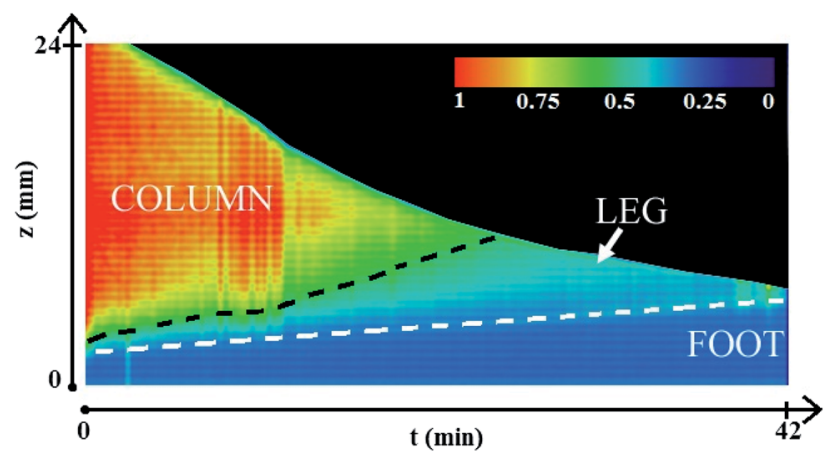

Fig. 7 Dependence of the normalized standard deviation $\sigma(r, t)$ of the intensity on the position $z$, plotted in false color along the vertical axis, as a function of $t$. The broken lines approximately identify the transition point where the leg merges into the foot (white) and with the overhanging column at $\phi_{0}$ (black). The scale on the top displays the color scale for the normalized standard deviation. 


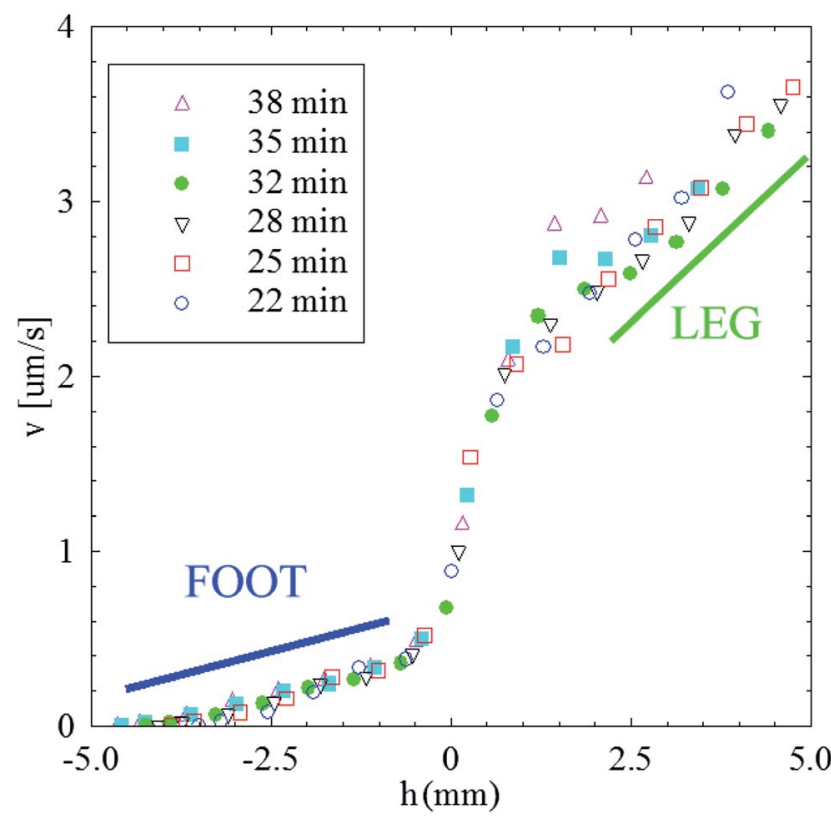

Fig. 8 Velocity profiles as a function of time and of the position in the cell, with the origin of the $z$-axis translated to the instantaneous position of the leg interface, as described in the text.

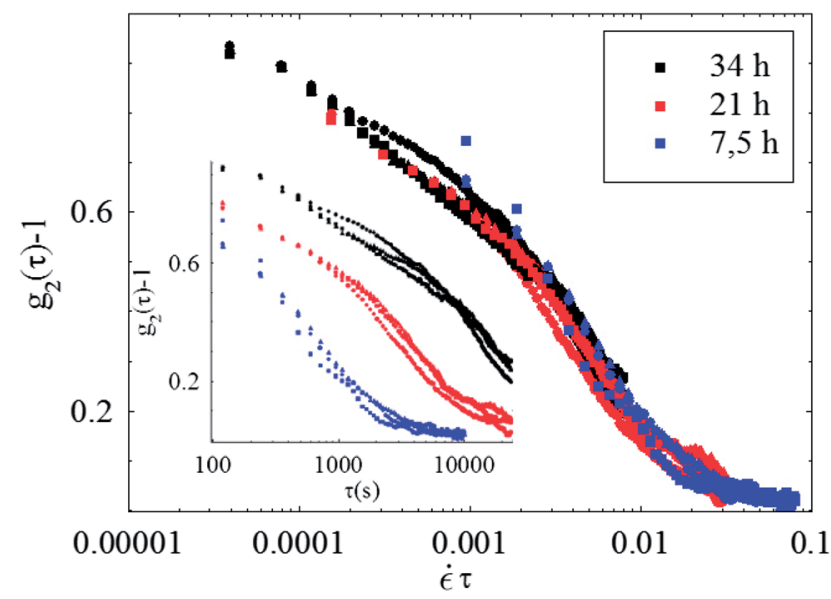

Fig. 9 Inset: correlation functions measured at three fixed heights $z=$ 0.8 (circles), 1.0 (squares), and $1.3 \mathrm{~mm}$ (triangles) and times (indicated in the legend) referring to the gel compaction stage. Main body: scaling of the same correlation function when plotted against $\dot{\varepsilon} \tau$.

\subsection{Microscopic dynamics during the compaction stage}

Further interesting information on gel compression can be obtained by PCI. Within this stage, indeed, the speckle dynamic is still sufficiently slow even at high $q$-vectors (namely, at the experimental scattering angle of $90^{\circ}$ ) to obtain PCI intensity correlation functions with sufficient accuracy, at least within the foot region. $\dagger \dagger$ The inset in Fig. 9 shows the excess correlation functions $g_{2}(\tau)-1$ obtained at three fixed heights $z$ from

† PCI also allows obtaining velocity fields within both the foot and the leg region, which are found in very good agreement with those found by GPV. the cell bottom at several times corresponding to the slow compaction stage. The gel ageing, witnessed by a progressive increase of the characteristic correlation time $\tau_{\mathrm{r}}$, defined as the time it takes for $g_{2}(\tau)-1$ to reduce by a factor $1 / e$, is not found to appreciably depend on $z$, and therefore on the local volume fraction. This distinctive feature of the ageing kinetics, which is consistent with previous findings, ${ }^{8}$ shows that the microscopic dynamics is fully ruled by the macroscopic strain rate $\dot{\varepsilon}$ which, as we have seen, is basically uniform within the foot region. Fig. 9 further supports this conclusion by showing that all correlation functions, when plotted against the rescaled time $\dot{\varepsilon} \tau$, collapse reasonably well on a single curve.

\section{Class B: creeping gels}

As we stated in Section 3, by increasing the initial concentration $\phi_{0}$ at fixed $\phi_{\mathrm{s}}$ the amorphous structures generated by the $\mathrm{L}-\mathrm{L}$ phase separation turn into a new class of gels that, after the quiescent period, compress smoothly, without showing any sign of the "catastrophic" collapse observed for class A gels. This drastic change in the settling kinetics fully takes places in a fairly narrow particle concentration region, and for values of $\phi_{0}$ that slightly decrease by increasing the quench depth. The kinetics of descent of the meniscus illustrative of the general behavior of these "creeping" gels is displayed in Fig. 10. We found that the time-dependence of the gel overall compression has actually a simple functional behavior. The inset in Fig. 10 shows indeed that, except in the very first stage, the fractional difference of the instantaneous gel height $h(t)$ from its asymptotic value at long time $h(\infty)$, normalized to the initial sample height $h(0)$, has a stretched-exponential dependence on time, $[h(t)-h(\infty)] / h(0) \sim \exp [-(t / \tau)]^{\alpha}$, with a stretching exponent $\alpha \simeq 1 / 2$. Notice also that, although the gel compression the

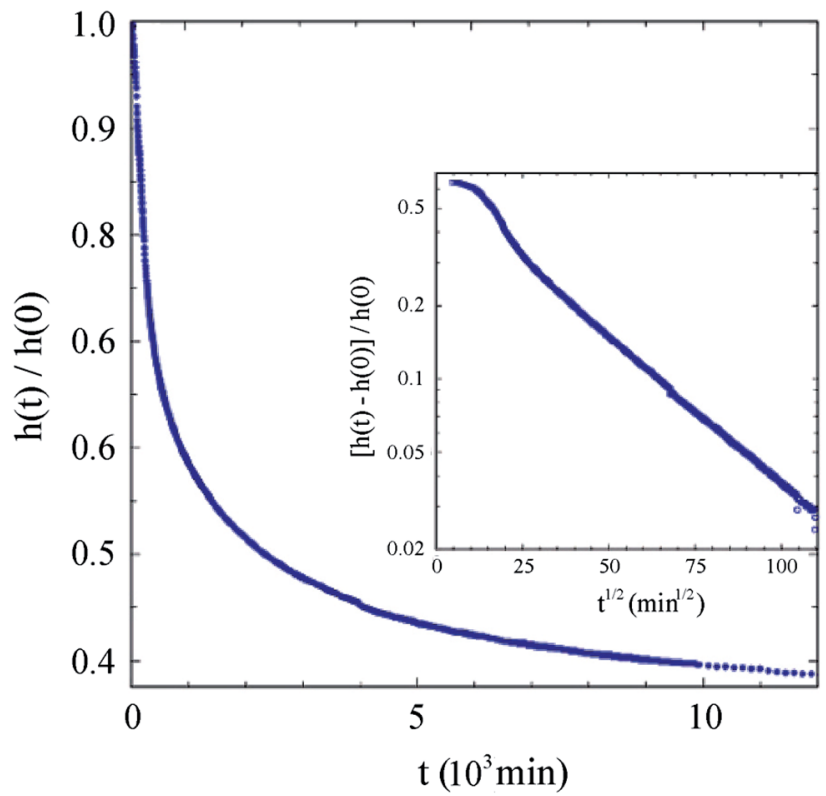

Fig. 10 Typical decrease of the overall sample height for a uniformly compressing gel, shown for a sample at $\phi_{0}=0.12, \phi_{\mathrm{s}}=0.14$. 
first couple of hours is very small, no sharp transition between a quiescent and a compressing stage is visible.

The distinctive compressive kinetics of these gels, which has often been discussed in the literature, ${ }^{4}$ cannot be easily accounted for by a simple poroelastic model. Very generally, the gel compression kinetics is fixed by the permeability $\kappa(\phi)$, relating the solvent back-flow through the gel to the pressure drop at boundaries, and by the effective compression modulus $K(\phi)$ controlling the mechanical response of the gel to stresses. In the standard poroelastic model, these two quantities are assumed to be time-independent and fixed only by the local volume fraction $\phi .{ }^{9}$ This implies that an initially uniform column at $\phi=\phi_{0}$, for which $\partial \phi / \partial z=0$, must settle at constant speed like a shock-wave, until it merges with the dense sediment (the foot) which progressively builds up at the cell bottom. Our data do not conversely display any initial settling stage at constant speed. This discrepancy with the predictions of the poroelastic model is not removed even if we speculate a consistent friction between the gel and the cell walls, for the latter would only yield a lower, but still constant, settling speed. The measurements we are going to present may shed some light on this challenging question.

\subsection{Concentration profiles}

The kinetic concentration profiles for class B gels are radically different from those we have formerly discussed. As shown in Fig. 11, the column is not uniform, and rather shows a slight but unmistakable inversion of the density profile, with values of $\phi$ which are larger on top than at the bottom of the column. Even more curious is that, at variance with any standard sedimentation process, the average volume fraction within the column is not constant, but increases with time. Both these peculiarities are present in the profiles of creeping gels described in ref. 4. This anomalous behavior, which is detected only during the first hours after sample preparation, apparently suggests that the gel, at least not too close to the sample top and bottom,

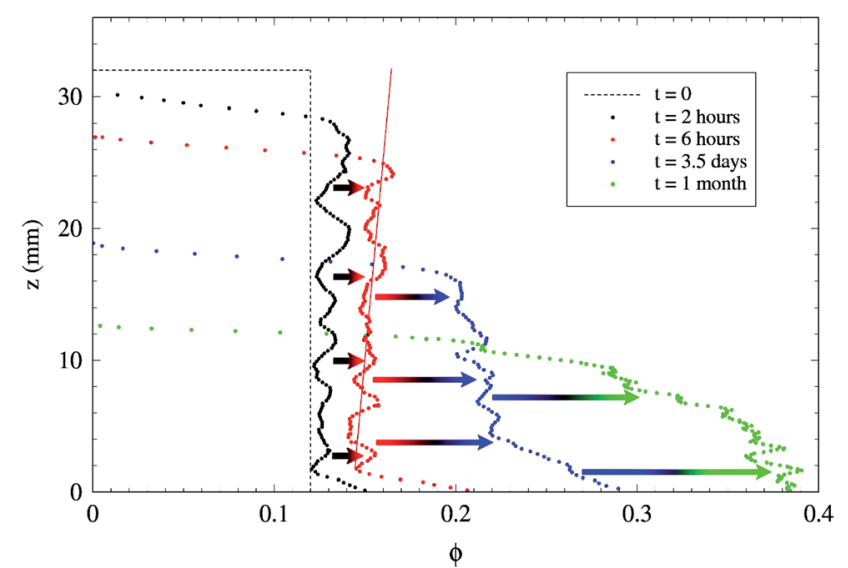

Fig. 11 Concentration profiles of a gel at $\phi_{0}=0.12, \phi_{\mathrm{s}}=0.14$ obtained at different times. The arrows show the progressive swelling of the column, while the inclined red line shows, in the first compression period, a slightly inverted density profile. shrinks uniformly, similar to what happens for polymer gels, ${ }^{31}$ with gravity playing little or no role. Later, the profile reverts to a more standard shape, with a local density monotonously decreasing with $z$, as expected for any gravity-driven sedimentation process. A tentative explanation of the apparent "syneresis" observed in the first stage is that, similarly to what happens for polymers, a newly-formed gel might be in a state of tension, with network strands that are overstretched with respect to their mechanical equilibrium condition (which is of course possible only for a gel generated by attractive interactions). In this scenario, gravity arguably has the sole effect of breaking the symmetry, favoring gel shrinkage along the vertical. Were this true, similar (but in this case isotropic) syneresis effects should be detected under microgravity conditions too. It is then useful to scrutinize whether this mechanisms may have repercussions on the dynamic behavior probed by GPV and PCI.

\subsection{Compression regimes}

For creeping gels, the GPV velocity profiles are fully laminar all along the compression stage. Fig. 12, which shows the $x$-averaged velocity profiles for several values of the lapse of time from sample preparation, allow distinguishing three time regimes:

1. For $t \leq 8 \mathrm{~h}$ (red curves), three regions can be set apart. Close to the cell bottom (region I), the settling speed increases exponentially with $z$. This is followed by a region at intermediate $z$ (region II) where $v_{z}$ increases linearly with $z$, so that the strain rate is approximately constant. Therefore, each volume element of the gel in region II, which corresponds to the column in the concentration profiles, compresses at the same rate. Mass conservation then implies that $\phi$ grows uniformly along the column, which must necessarily "swell" as a whole, as observed. On the very top of the column, we find another region (III) that also displays an almost constant strain rate $\dot{\varepsilon}_{\text {III }}$, which is however larger than the strain rate $\dot{\varepsilon}_{\text {II }}$ in the region below. Because of this, an excess of particles may accumulate on top of

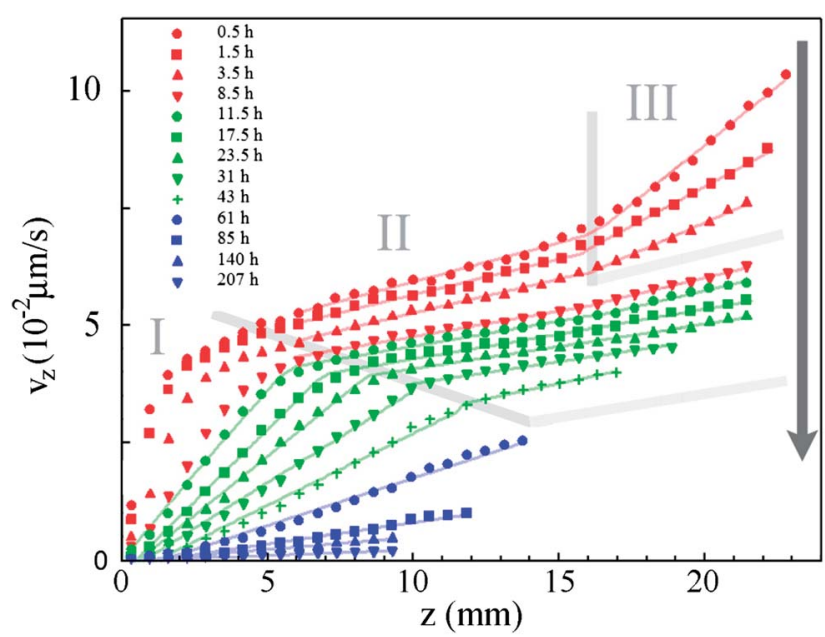

Fig. 12 Velocity profiles for a gel at $\phi_{0}=0.12, \phi_{\mathrm{s}}=0.12$, for several values of $t$ between $1 \mathrm{~h}$ and 10 days from sample preparation, with $t$ increasing in the arrow direction. 
the slower-settling region II, consistently with the observation of a density inversion in the profile in the initial stage of the settling process.

2. In less than 3 hours, however, regions III merges into region II, while the strain rate attains a constant value $\dot{\varepsilon}_{\mathrm{I}}$ in region I too, similarly to what is found for the gravity-driven compaction of class A gels (green curves). Since $\dot{\varepsilon}_{\mathrm{I}}>\dot{\varepsilon}_{\mathrm{II}}$, the profile progressively reverts to a standard shape, and the sample gets more concentrated at the bottom.

3. As time goes by, region II progressively shrinks, eventually disappearing for $t \gtrsim 60 \mathrm{~h}$. The final compression stage is then described by a single value of the strain rate, which decreases with time (blue curves).

The GPV velocity profiles provide then an explanation of how the peculiar feature of the concentration profiles generate. When re-examined in a different fashion, however, they also support the conjecture we made concerning the presence of syneresis effects. To see this, let us consider the time-evolution of the settling velocity at a fixed distance from the cell bottom, shown in Fig. 13. In the lower sample region $(z=4 \mathrm{~mm})$, the whole time dependence can be fitted by a single exponential, whose time constants $\tau_{1}$ increases with $z$. Conversely, the curve for $z=11 \mathrm{~mm}$ shows a first approximately exponential decay, followed by a second exponential relaxation that starts after the first one has almost reached a plateau value $v_{\mathrm{p}}$ of about $65 \%$ of the initial settling speed and eventually decays to $v=0$. A similar behavior is common to the whole upper sample region $(z \gtrsim 6 \mathrm{~mm})$. As shown in the inset of Fig. 13, whereas the fitted decay constant $\tau_{\mathrm{u} 1}$ of the first exponential does not depend on $z$, both the decay constants $\tau_{\mathrm{u} 2}$ of the second exponential and the values of $\tau_{1}$ increase linearly with $z$, and moreover fall on a single straight line. This strongly suggests that $\tau_{1}$ and $\tau_{\mathrm{u} 2}$ witness the same, gravity-driven compression regime (regime G in Fig. 13).

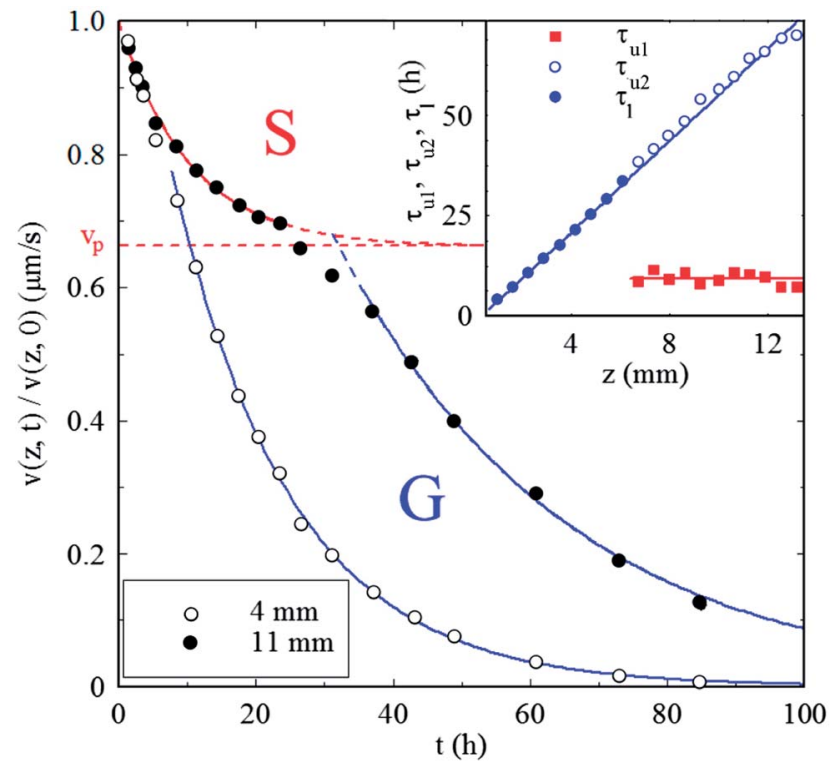

Fig. 13 Body: time-dependence of $v_{z}(t, z)$ for two different distances $z$ from the cell bottom, indicated in the legend. Inset: values of $\tau_{l}$ (blue full dots), $\tau_{\mathrm{u} 2}$ (blue open dots) and $\tau_{1}$ as a function of $z$.
Conversely, the lack of dependence of $\tau_{\mathrm{u} 1}$ on $z$ is suggestive of a very different mechanism governing the initial compression stage, which is not ruled by gravity (regime $S$ ). The larger the fractional contribution $\Delta_{\mathrm{S}}=1-v_{\mathrm{p}}$ of this mechanism is, the closer the investigated region is to the top of the gel. We actually found that, in the region $z \geq 6 \mathrm{~mm}, \Delta_{\mathrm{S}}$ increases almost linearly with $z$ (data not shown), which would imply a constant strain rate within regime $\mathrm{S}$. This is in fact also supported by the linear behavior of $v_{z}(z)$ in region II of the data in Fig. 12 obtained for $t \lesssim 30-40 \mathrm{~h}$. Such a uniform compression suggests that regime $S$ could be actually identified with the initial time lapse during which a spontaneous "syneresis" process takes place.tt

\subsection{Microscopic dynamics for compacting gels}

Compaction of class B gels is sufficiently slow to be investigated by PCI during the whole restructuring stage, which we followed up to more than 10 days. The speckle pattern image was subdivided into blocks with a height of $100 \mathrm{px}$ and laterally extending over the whole width of the investigated sample region ( $W=400 \mathrm{px})$. The progressive change in the microscopic dynamics can be quantitatively analyzed by means of the correlation functions $g_{2}(\tau)$, using a coarse-graining time interval of $\Delta t=30 \mathrm{~min}$. All excess correlation functions $g_{2}(\tau)-1$ eventually decay to zero regardless of the aging time, showing that the gel dynamics is never fully arrested: namely, the gel slowly "creeps" even over macroscopic time scales. The aging behavior of these gels is better understood by recalling that for class A gels, where the microscopic dynamics is fully ruled by the macroscopic strain rate, the characteristic time scale $\tau_{\mathrm{r}}$ over which the correlation functions decay to $1 / e$, when regarded as a function of the instantaneous strain rate at the same location, scale as $\tau_{\mathrm{r}}(\dot{\varepsilon}) \propto \dot{\varepsilon}^{-1}$, and therefore the cumulative strain over a microscopic relaxation time, $\varepsilon=\dot{\varepsilon} \tau_{\mathrm{r}}(\dot{\varepsilon})$, does not depend on $\dot{\varepsilon}$. For class B gels, the dependence of $\varepsilon$ on the strain rate turns out to be radically different in the two compression regimes highlighted in Section 5.2. Panel A in Fig. 14, where the cumulative stress is plotted for three different averaging boxes located at the bottom $(z=2.5 \mathrm{~mm})$, in the middle $(z=6 \mathrm{~mm})$, and at the top of the gel $(z=12 \mathrm{~mm})$, shows that in the later-stage regime $\mathrm{G}$ the microscopic dynamics of the gels is again controlled by $\dot{\varepsilon}$, and that the cumulated strain attains a limiting value $\varepsilon \simeq 3.5 \times$ $10^{-3}$ which does not depend on $z$ and is actually comparable to the typical values measured in colloidal systems ${ }^{\mathbf{8}, 32}$ or molecular glass formers. ${ }^{33}$ In regime S, conversely, the microscopic relaxation time does not appreciably depend on the strain rate. Hence, in the initial stage, when the compression kinetics resembles a syneresis process, the microscopic dynamics is basically unrelated to the macroscopic compression rate. As a matter of fact, Panel B in Fig. 14 shows that not only $\tau_{\mathrm{r}}$, but also the whole correlation functions obtained in the first compression regime (which, we recall from Section 5.2, pertains only to

\#t This behavior actually resembles what we expect for a stretched rubber band constrained at one end that, in the presence of viscous overdamping, relaxes exponentially to equilibrium with a local speed that grows linearly with the distance from the fixed end. 

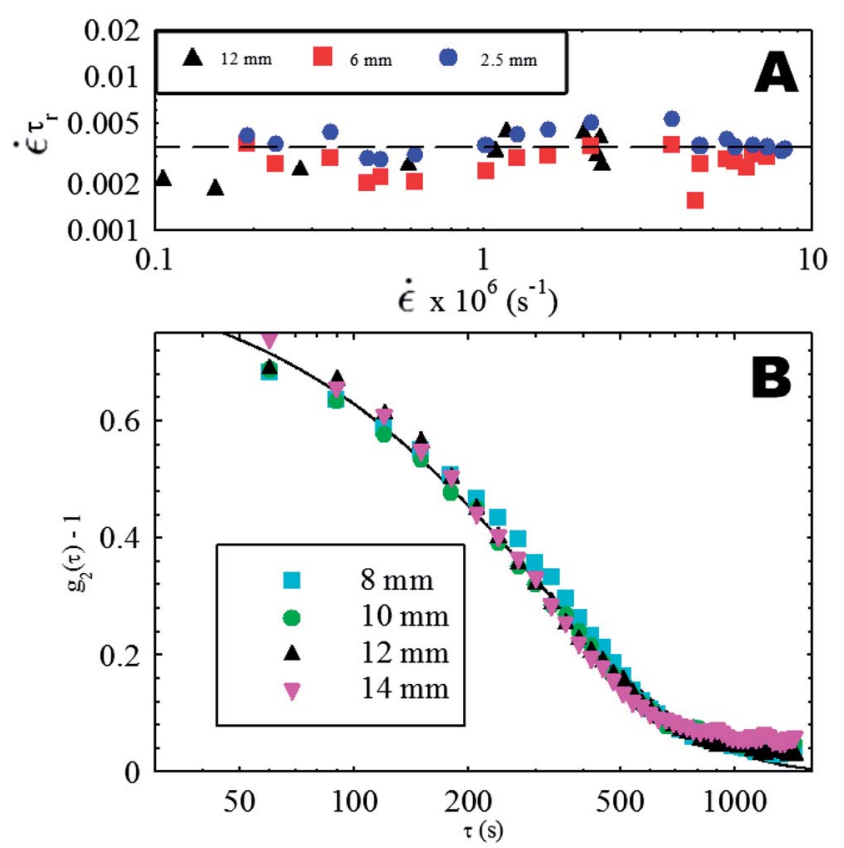

Fig. 14 Panel A: cumulative strain $\varepsilon=\dot{\varepsilon} \tau_{\mathrm{r}}$ versus the strain rate $\dot{\varepsilon}$ for a class B gel at $\phi_{0}=0.12$ and $\phi_{\mathrm{s}}=0.12$, obtained in regime $G$ within the averaging boxes indicated in the legend. The broken line shows that, at late stage, the same strain-rate independent value $\varepsilon \simeq 0.0035$ is attained all over the gel. Panel B: correlation functions obtained in regime $\mathrm{S}$ ( $6 \mathrm{~h}$ after sample preparation) for different values of $z$, fitted with a single exponential.

the upper part of the cell) do not appreciably depend on position $z$.

Fig. 14B also shows that, during the early compression stage, the correlation functions are reasonably well fitted by a single exponential decay. However, as the gel gets progressively more compressed, the correlation functions change progressively. Fig. 15, which refers to the very late stage of compaction $(t>$ $100 \mathrm{~h}$ ), actually shows that their shape is noticeably different depending on whether we consider the less concentrated gel in the upper part of the cell, or the very compacted region close to the cell bottom. Whereas for $z>8 \mathrm{~mm}$, where the local volume
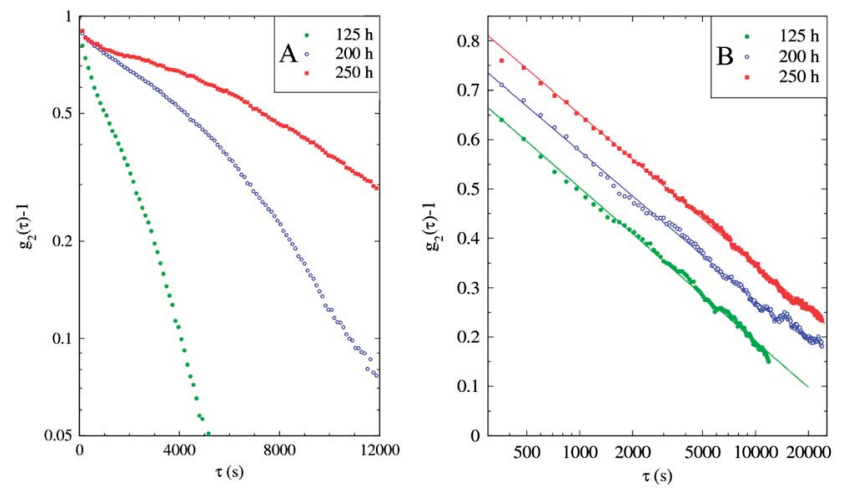

Fig. 15 Correlation functions obtained at a very late compression stage in the upper part of the sediment ( $z=100 \mathrm{~mm}$, Panel A) and close to the cell bottom ( $z=2 \mathrm{~mm}$, Panel B). fraction does not exceed $\phi \simeq 0.38$, the correlation functions are not drastically different from exponentials (Panel A), for $z<8$ $\mathrm{mm}$ they start displaying a consistent long-time tail. In particular, if we focus on the region very close to the cell bottom $(z=2$ $\mathrm{mm}$, Panel B), where $\phi$ attains a value as large as $0.40, g_{2}(\tau)$ display an evident logarithmic decay over more than two decades in $\tau$. Logarithmic decays of this kind are a distinctive tract of the attractive colloidal glasses forming, for colloids interacting via short-range interactions, outside the $\mathrm{L}-\mathrm{L}$ coexistence region for rather larger values of $\phi .^{34-38}$ It is then tempting to assimilate the highly compressed depletion gels that are generated by gravitational settling to this kind of amorphous structures. Further experiments, performed at different values of $\phi_{0}$ and $\phi_{\mathrm{s}}$, are however required to validate this ansatz.

\section{Class C: crystallizing gels}

For shallow quenches (namely, for a $\tau$ parameter $0.002 \lesssim \tau \lesssim$ 0.01 ), the depletion gels that form by arrested spinodal decomposition undergo a totally different time evolution. Depending on $\phi_{0}$, they may (open triangles in Fig. 2) or may not (full triangles) show clear evidence that a collapse, similar to those observed for class A gels, takes place. However, in both cases they start to host, on a time scale ranging of tens of minutes, a small number of tiny compact objects, which are barely visible with the naked eye.\$§ Although the MFA particles we use are too small to display Bragg peaks in a colloidal crystal phase, these objects, which in a transmission imaging geometry appear as little dark spots on a brighter background (see inset (a) in Fig. 16) and whose motion can be followed by particle tracking, may reasonably be assumed to consist of little crystallites. This conclusion is supported by the later evolution of the system, which we specifically describe for a suspension prepared at $\phi_{0}=0.08, \phi_{\mathrm{s}}=0.10$. During an initial stage lasting for a few hours, most of the crystallites look as frozen inclusions trapped in a solid amorphous matrix. Yet, at random instants, some of them suddenly break free from the matrix and start performing a rather erratic motion, which sometimes shows puzzling effects of motion directed against gravity. The total number of crystallites grows in time until, about $10 \mathrm{~h}$ after sample preparation, the gel suddenly breaks apart, and the crystallites rapidly settle to the cell bottom (see inset (b) in Fig. 16) merging into a single dense phase that, in a couple of hours, takes up about $10 \%$ of the total amount of MFA particles in the sample (body of Fig. 16). This accumulation process goes on at a much slower and approximately constant rate for the next $50 \mathrm{~h}$. In this stage a barely visible meniscus is visible. After $50 \mathrm{~h}$ the growth of the dense phase slows down to reach the final height after a month.

The very dense phase at the cell bottom that eventually forms does not show any evidence of compaction over a time scale of the order of a month. A rough evaluation of the particle volume

$\S \S$ For the systems indicated with open triangles in Fig. 2, this approximately happens when the average volume fraction of the sediment is about 0.08 , corresponding to lower value of $s$ where crystal nucleation is seen to occur before any appreciable settling of the gel takes place (full triangles in Fig. 2). 


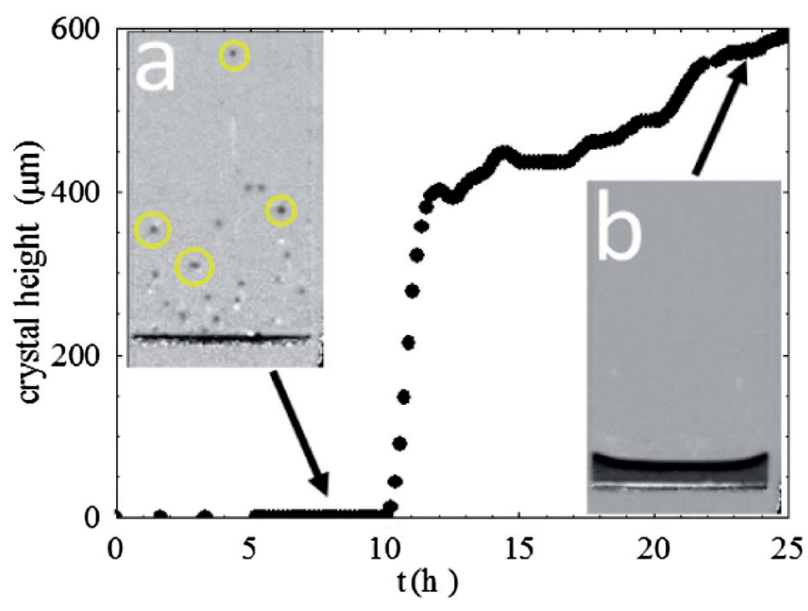

Fig. 16 Body: growth in time of the crystalline phase in a sample at $\phi_{0}$ $=0.08, \phi_{\mathrm{s}}=0.10$. Inset (a) shows a transmission image of the sample before the crystallites, appearing as dark spots, accumulate and merge at the cell bottom (a few crystallites are evidenced by yellow circles), whereas the single crystal that eventually builds up appears as a dark band in the inset (b).

fraction of the sediment, obtained from its final height and from $\phi_{0}$ assuming that the supernatant is fully devoid of particles, yields $\phi \gtrsim 0.69 \pm 0.02$, which is very close to the ordered close packing value $\phi_{\text {ocp }}=0.74$ for hard spheres. This is fully confirmed by more accurate DeLS measurements, yielding an almost uniform concentration profile with $\phi=0.72 \pm 0.02$, in agreement with what has been found in previous investigations. $^{14}$

For short-ranged attractive potentials, the rate of crystal nucleation is predicted to strongly increase by approaching the metastable $\mathrm{L}-\mathrm{L}$ coexistence curve even in the homogenous (but metastable) suspension region, and this is actually seen in protein crystallization. ${ }^{39,40}$ Yet, this takes place only for concentrations close to or higher than the critical point value $\phi_{\mathrm{c}}$ $\simeq 0.26$, which are far larger than those investigated in this work. In fact, we have never detected any crystal nucleation before the sample is quenched within the $\mathrm{L}-\mathrm{L}$ coexistence gap. Nevertheless, we have seen that, provided that the quench into the L-L gap is sufficiently shallow, crystallization is enhanced in a pre-formed gel phase too, which acts as a "cradle" for the nucleation and growth of crystallites.

That L-L phase separation may strongly enhance crystal nucleation has also been recently reported for a colloid/polymer mixture with a much larger size ratio $\delta \simeq 0.52$ (actually yielding a L-L phase separation which is stable with respect to crystallization), provided the suspension is quenched within the threephase (colloidal gas, liquid, and solid) coexistence region. ${ }^{13} \mathrm{Just}$ after homogenization, these samples show a rapid increase of the turbidity, highlighting an initial phase separation into a colloidal gas and a colloidal liquid. In normal gravity, these two phases are driven apart, and after a few hours crystals appear within the bulk of the colloidal-rich phase and slowly settle to the cell bottom. However, under microgravity conditions, the two fluid phase does not physically segregate, and the crystallites that nucleate within the colloidal liquid may grow to the point that they form a disordered network (a "crystal gel") that hinders further coarsening of the domains. $\uparrow$ I It is however important to point out that, both in earth and in space experiments, crystal formation nevertheless takes place within a fluid phase. Our observations are then, to our knowledge, the first evidence of a full conversion into a colloidal crystal of a preexisting gel phase. We may wonder whether, in the absence of gravitational stresses leading to the collapse of the host gel matrix, also the crystallites we found may organized into a kind of weak "crystal gel". As a matter of fact, we plan to investigate this issue in microgravity experiments already planned in the framework of the ESA International Project "Solidification of Colloids in Space".

\section{Discussion}

This investigation has shown how the extensive application of novel optical correlation methods, together with a careful mapping of the investigated samples onto the system phase diagram, may allow obtaining a comprehensive view of the behavior under gravity of depletion gels generated by arrested phase separation within a metastable L-L gap.

Several issues concerning the observed kinetics, and related in particular to the occurrence of the anomalous "leg" feature detected in the settling profiles of class A gels, and to the very peculiar early-stage compression behavior of class B gels, are however still very open. These two structures may actually be rather intimately related. In fact, the concentration values at which the leg feature forms, shown as open dots in Fig. 2, lie very close to the borderline between class A and B gels. Moreover, Fig. 5 shows that, before merging into the foot, the leg shows a progressive uniform expansion quite similar to what is evidenced in Fig. 11 for creeping gels. Finally, the settling velocity within the leg region is smooth and laminar like for creeping gels, although consistently faster. It is then tempting to speculate that the leg may be just a "transient manifestation" of a creeping gel, occurring as a kind of dynamic transition in the settling kinetics of a collapsing gel when its local volume fraction exceeds the value marking the boundary with class B gels. However, this assumption requires further supporting evidence, since it is unlikely that the way the leg builds up (by accumulation of fragmented clusters) leads to a structure closely resembling class B gels, which are fully interconnected networks right from the start.

A very peculiar feature of class B gels is the occurrence of an initial stage when the restructuring kinetics is not apparently ruled by the gravitational stress, but rather by an internal mechanism resembling those driving syneresis in polymer gels. These preliminary observations, if confirmed by a more detailed investigation, would suggest that, at sufficiently high particle

ๆ This strongly reminds us of the sol-gel transition occurring in undercooled paraffin mixtures, where similar "crystal gels" form because of short-range attractive forces between micron-size wax crystallites suspended in a lower molecular weight hydrocarbon fluid, leading to the occurrence of a "pour point" temperature, below which crude oil no longer flows. ${ }^{41}$ 
volume fraction, depletion gels may form in a state of "internal tension", which relaxes during the initial evolution stage before gravity settling effects become relevant. Another issue that deserves further investigation is the occurrence of a logarithmic relaxation in the very dense sediment resulting from the compaction over long times of class B gels, which may suggest that the distinction between this structure and a colloidal glass, located close to the point where the attractive and repulsive glass lines meet, may be more nominal than substantial, at least for what concerns the microscopic dynamics.

To inquire about the structural difference between the kinds of gels we found, and about the evidence of a region within the $\mathrm{L}-\mathrm{L}$ gap where the original gels act as cradles for crystal nucleation, a comparison with the numerical results by Fortini et $a .^{12}$ is particularly useful. Although the latter refer to a depletion system with quite a larger size ratio $(\delta=0.15)$, the non-equilibrium phase diagram resulting from Brownian dynamics simulations (see Fig. 8 in ref. 12) displays several general features in common with the present investigation. Indeed, for sufficiently high interaction strength $U / k_{\mathrm{B}} T$, and strictly within L-L phase coexistence, two kinds of dynamically arrested phases (there named "attractive glasses", rather than gels) can clearly be set apart. These two structures are separated by a rather sharp boundary B that, although dubbed "percolation line", should not be confused with the usual dynamic percolation threshold, bounding the region where the system contains a sample-spanning infinite cluster.||| Rather, line B separates structures which are radically different in terms of kinetics of formation and mesoscopic morphology. Attractive glasses on the right side of $\mathrm{B}$ attain right from the start the structure of a percolating network. On the left side, conversely, the phase separation process first generates large, but finite size clusters, which eventually, but only over much longer time scales, stick to each other forming an arrested structure. As a matter of fact, evidence of arrested structures made of interconnected finite-size clusters with a fractal structure was found in early measurements of gelation in colloid + surfactant systems at low particle concentration. ${ }^{42}$ We are currently investigating by near-field scattering methods whether, during the delay time preceding gravitation collapse or compression, the structure of the two gels that initially form can be related to those found in numerical simulations.

The same numerical result, however, shows that at lower $U /$ $k_{\mathrm{B}} T$, crystallization strongly competes with spinodal decomposition: nucleation starts within the denser phase before the systems coarsen into macroscopic phase domains, and the systems eventually freeze into structures which resemble the "crystal gels" found by Sabin et al. under microgravity conditions. ${ }^{13}$ A strong enhancement of the nucleation rate close to the coexistence curve with respect to the one-phase region is fully consistent with what we found. Yet, our results suggest that, for very-short range depletion, dynamic arrest may actually come ahead without, however, stopping crystal growth, so that the whole gel eventually turns into an ordered structure. This final

||| In the AHS model, the latter includes almost the whole coexistence region. evidence surely calls for a more detailed investigation of the effect of the interaction range on the competition between nucleation and phase separation.

\section{Acknowledgements}

We thank L. Cipelletti, W. Poon, E. Zaccarelli, and M. Hermes for fruitful discussions and comments. This work has been supported by the Italian Ministry for Education, University, and Research (MIUR) through PRIN 2012 and "Futuro in Ricerca" (Project Anisoft/RBFR125H0M) funding.

\section{References}

1 M. Dijkstra, J. M. Brader and R. Evans, J. Phys.: Condens. Matter, 1999, 11, 10079.

2 H. N. W. Lekkerkerker and R. Tuinier, Colloids and the Depletion Interaction, Springer, Heidelberg, 2011.

3 E. Zaccarelli, J. Phys.: Condens. Matter, 2007, 19, 323101.

4 L. Starrs, W. Poon, D. Hibberd and M. Robins, J. Phys.: Condens. Matter, 2002, 14, 2485.

5 A. Fluerasu, A. Moussaid, A. Madsen and A. Schofield, Phys. Rev. E: Stat., Nonlinear, Soft Matter Phys., 2007, 76, 010401.

6 J. Lietor-Santos, C. Kim, M. Lynch, A. Fernandez-Nieves and D. Weitz, Langmuir, 2009, 26, 3174.

7 L. J. Teece, M. A. Faers and P. Bartlett, Soft Matter, 2011, 7, 1341.

8 G. Brambilla, S. Buzzaccaro, R. Piazza, L. Berthier and L. Cipelletti, Phys. Rev. Lett., 2011, 106, 118302.

9 S. Buzzaccaro, E. Secchi, G. Brambilla, R. Piazza and L. Cipelletti, J. Phys.: Condens. Matter, 2012, 24, 284103.

10 P. Bartlett, L. J. Teece and M. A. Faers, Phys. Rev. E: Stat., Nonlinear, Soft Matter Phys., 2012, 85, 021404.

11 I. Zhang, C. P. Royall, M. A. Faers and P. Bartlett, Soft Matter, 2013, 9, 2076-2084.

12 A. Fortini, E. Sanz and M. Dijkstra, Phys. Rev. E: Stat., Nonlinear, Soft Matter Phys., 2008, 78, 041402.

13 J. Sabin, A. E. Bailey, G. Espinosa and B. J. Frisken, Phys. Rev. Lett., 2012, 109, 195701.

14 S. Buzzaccaro, R. Rusconi and R. Piazza, Phys. Rev. Lett., 2007, 99, 098301.

15 V. Degiorgio, R. Piazza, T. Bellini and M. Visca, Adv. Colloid Interface Sci., 1994, 48, 61.

16 R. Piazza, V. Degiorgio, M. Corti and J. Stavans, Phys. Rev. B: Condens. Matter Mater. Phys., 1990, 42, 4885-4888.

17 L. Cipelletti, G. Brambilla, S. Maccarrone and S. Caroff, Optic. Express, 2013, 21, 22353.

18 L. Cipelletti, H. Bissig, V. Trappe, P. Ballesta and S. Mazoyer, J. Phys. Condens. Matter., 2003, 15, S257.

19 E. Secchi, T. Roversi, S. Buzzaccaro, L. Piazza and R. Piazza, Soft Matter, 2013, 9, 3931.

20 R. Piazza, in Colloidal Foundations of Nanoscience, ed. Elsevier, Elsevier, Amsterdam, 2004, ch. 8.

21 M. Giglio, M. Carpineti, A. Vailati and D. Brogioli, Appl. Opt., 2001, 40, 4036.

22 R. Cerbino and V. Trappe, Phys. Rev. Lett., 2008, 100, 188102. 
23 S. Buzzaccaro, E. Secchi and R. Piazza, Phys. Rev. Lett., 2013, 111, 048101.

24 M. A. Miller and D. Frenkel, J. Chem. Phys., 2004, 121, 535.

25 F. Cardinaux, T. Gibaud, A. Stradner and P. Schurtenberger, Phys. Rev. Lett., 2007, 99, 118301.

26 K. N. Pham, A. M. Puertas, J. Bergenholtz, S. U. Egelhaaf, A. Moussaïd, P. N. Pusey, A. B. Schofield, M. E. Cates, M. Fuchs and W. C. K. Poon, Science, 2002, 296, 104.

27 S. Buzzaccaro, A. Tripodi, R. Rusconi, D. Vigolo and R. Piazza, J. Phys.: Condens. Matter, 2008, 20, 494219.

28 S. Manley, J. Skotheim, L. Mahadevan and D. Weitz, Phys. Rev. Lett., 2005, 94, 218302.

29 J.-M. Condre, C. Ligoure and L. Cipelletti, J. Stat. Mech. Theor. Exp., 2007, 2007, P02010.

30 A. Duri, D. Sessoms, V. Trappe and L. Cipelletti, Phys. Rev. Lett., 2009, 102, 085702.

31 M. Doi, J. Phys. Soc. Jpn., 2009, 78, 19.

32 R. Besseling, E. R. Weeks, A. B. Schofield and W. C. K. Poon, Phys. Rev. Lett., 2007, 99, 028301.
33 R. A. Riggleman, H. N. Lee, M. D. Ediger and J. J. de Pablo, Phys. Rev. Lett., 2007, 99, 215501.

34 F. Mallamace, P. Gambadauro, N. Micali, P. Tartaglia, C. Liao and S. Chen, Phys. Rev. Lett., 2000, 84, 5431.

35 X. Lu, S. Mochrie, S. Narayanan, A. Sandy and M. Sprung, Phys. Rev. Lett., 2008, 100, 045701.

36 X. Lu, S. Mochrie, S. Narayanan, A. Sandy and M. Sprung, Soft Matter, 2010, 6, 6160.

37 A. M. Puertas, M. Fuchs and M. E. Cates, Phys. Rev. Lett., 2002, 88, 098301.

38 E. Zaccarelli, I. Saika-Voivod, S. V. Buldyrev, A. J. Moreno, P. Tartaglia and F. Sciortino, J. Chem. Phys., 2006, 124, 124908.

39 P. R. ten Wolde and D. Frenkel, Science, 1997, 277, 1975.

40 R. Piazza, Curr. Opin. Colloid Interface Sci., 2000, 5, 38.

41 E. Vignati, R. Piazza, R. F. G. Visintin, R. Lapasin, P. D'Antona and T. P. Lockhart, J. Phys.: Condens. Matter, 2005, 17, S3651.

42 R. Piazza and G. Di Pietro, Europhys. Lett., 1994, 28, 445. 